Journal of Nepal Mathematical Society (JNMS), Vol. 2, Issue 2 (2019); J. Kafle, P. Kattel

\title{
Simulations for Flow-Symmetry Through r.avaflow in the General Two-Phase Mass Flow Model
}

\author{
Jeevan Kafle ${ }^{1,2}$, Parameshwari Kattel ${ }^{1,3}$ \\ ${ }^{1}$ Department of Mathematics, School of Science, Kathmandu University, Dhulikhel, Kavre, Nepal. \\ ${ }^{2}$ Central Department of Mathematics, Tribhuvan University, Kathmandu, Nepal. \\ ${ }^{3}$ Department of Mathematics, Tri-chandra Multiple Campus, Tribhuvan University, Kathmandu, Nepal. \\ Correspondence to: Parameshwari Kattel, Email: pkattel@student.edu.ku.np
}

\begin{abstract}
Gravitational flows, e.g., landslide, debris flow and avalanches are hazardous mass wasting processes. The proper understanding of their dynamics is very important. As laboratory experiments can not perfectly model their initiation process and field assess of the live events are very difficult, numerical experiments have become the promising way for the study of their flow dynamics. Here we employ the enhanced version of two-phase mass flow model 33 through the open source computational code, r.avaflow to analyze the issue of symmetry in the flow. Two-phase debris mass are triggered from all the flanks of the three different pyramids (triangle-based, square-based and octagon-based) with different rotational symmetry and study the flow pattern along with maximum kinetic energy of the flow. Flow past two different types of obstacles (a tetrahedron and a square based pyramid) are also observed. The possible causes of asymmetry in the flow are also analyzed.
\end{abstract}

Keywords: Debris flows, Two-phase mass flows, r.avaflow, Rotational symmetry, Asymmetry, Flowobstacle-interaction

\section{Introduction}

Geophysical mass flows/movements like landslides, debris flows, rock falls, snow avalanches, flash floods and tsunamis devastates natural landscapes and infrastructures, and claim human lives [8, 45. The study of their triggering factors and dynamics are very important for the mitigative and preventive measures. The study of their dynamics during the events is very difficult due to the lack of the appropriate knowledge and techniques to predict the events accurately and also due to the rapid motion of the huge mass creating high momentum [42. As the study in laboratory cannot perfectly model the natural events and also there is high level of difficulty in scaling, the numerical experiments, or computer simulations have become the most convenient way for the study of the geophysical mass flows/movements [4, 41. Not only the efficiency of the new generation computers, but also the emerging numerical methods and computational techniques have made the simulations more accurate and much faster than the previous attempts [1, 15, 25, 29, 39]. There are some open source codes for computational fluid dynamics, including, OpenFOAM, which has a wide range of features to solve complex fluid flows related to chemical reactions, turbulence and heat transfer, acoustics, solid mechanics and electromagnetics 2. Although many sophisticated concepts and techniques exist for modeling the motion of multi-phase gravitational flows, most of them cannot properly address the complexity of the processes or process chains 1 .

As the evolving behaviour of debris flow is complex with different types of solid constituents with viscous fluid, a single rheological model equation might not be sufficient for its accurate description. Different rheological models were proposed and employed in the past, but most of them were effectively single-phase models 13 . Some significant research activities in the field of debris flows and similar gravitational mass flows in the past few decades include (i) single-phase dry granular avalanches [11, 12, 32, 36, (ii) singlephase debris flows [3, 5, 37, (iii) mixture flows [13, 14, (iv) two-fluid debris flow [30, and (v) a two-layered model 9 .

The general two-phase mass flow model 33 has addressed the complex fluid-solid interactions and includes different dominant aspects of two-phase mass flows, namely the enhanced non-Newtonian viscous stress, virtual mass and drag. Employing the general two-phase mass flow model [33], many realistic simulations have been carried out to simulate the real field events and also with generic topographies. Kattel [21] presented the evolution of solid, fluid and the total debris bulk for different geometric and material parameters. Kattel et al. 22 simulated the flow similar to glacial lake outburst floods in three different 
initial and boundary conditions. Employing the general two-phase mass flow model [33, the interaction of two-phase landslide with still reservoir in geometrically two dimension was simulated by Pudasaini 34] to show the explicit evolution of the solid and fluid phases and the produced tsunami waves at impact [16]. For idealized geometry, Kafle [17] and Kafle et al. [18] advanced further by simulating two-phase and geometrically three-dimensional flows impacting a fluid reservoir and presented some observable natural phenomena like, the splash, tsunami generation, and submarine mass movements. The results in Kafle et al. 18 revealed that the submerge time scaling for a deformable two-phase landslide differ substantially from the non-deformable solid. Kafle and Tuladhar 19 presented and discussed a simulation related to a partially submerged landslide in a fluid reservoir employing the two-phase mass flow model 33 to observe the explicit evolution and propagation of surface tsunami waves, and solid waves as submarine mass movement.

Ostacle interactions are mostly discussed for granular flows [6, 38, 40, 43, or for a debris bulk [7, 31, 44] without considering two separate phases. Kattel et al. 24] performed a number of computational experiments to present detailed dynamical interactions of the flowing debris mass with stationary tetrahedral obstacles mounted on a generic slope topography that merges to horizontal run out plane. They described the run out scenarios for solid and fluid phases separately and for a debris bulk as a whole for tetrahedral obstacles. Employing the same model, Kattel and Tuladhar 23] performed numerical experiments on obstruction and contraction ratio of two-phase debris flow due to converging lateral shear walls. Kafle et al. 20. presented first-ever three-dimensional, high-resolution simulation results for a two-phase landslide impacting a fluid reservoir installed with obstacles of different shapes, sizes, numbers, and at different positions on the subaerial slopes and bathymetry.

Recently developed open source code, r.avaflow can use suitable single- and advanced two-phase mass flow models according to the nature of the flow addressing the role of the pore fluid; includes entrainment and deposition of component phases involved in the flow; can use several appropriate and realistic basal friction models; employs a high-resolution numerical scheme for flow over natural topography [1]. With these features, r.avaflow has aimed at creating a free software supporting experts and professionals to manage geophysical mass flow hazards, and distributing a user-friendly, freely accessible, GIS-based open source simulation tool. The tool contains built-in functions for visualizing the simulation results without the need of any post processing of the numerical results 1 .

r.avaflow has been developed along two lines, for two specific group of users. (i) r.avaflow [EXPERT] is for the researchers who wish to pursue parameter studies or some computational experiments at a high level of complexity. This version is designed as a module of GRASS GIS 7 and also employs the R Project for statistical computing, allowing for parallel processing. (ii) r.avaflow [PROFESSIONAL] is targeted for the practitioners, who wish to conduct backward- or forward-calculations of particular mass flows. Although it serves with a user-friendly interactive visualization function, it is not designed for complex computational experiments that includes multiple model runs [1].

To test the functionalities of r.avaflow [1], Mergili et al. [26] conducted two sets of experiments employing the general two-phase mass flow model [33]: (i) as a generic process chains consisting in bulk mass and hydrograph release into a reservoir with entrainment of the dam and impact downstream (ii) as the back analysis of the prehistoric Acheron rock avalanche of New Zealand, with optimization of key parameters. Mergili et al. 27] back-calculated a geological event, where a landslide from a moraine slope triggered a multi-lake outburst flood in the Artizón and Santa Cruz valleys, Cordillera Blanca, Peru (2012) with r.avaflow. The reconstructed flow patterns largely matched with the documented geomorphologic changes induced by the event. Mergili et al. [28] used r.avaflow, and the two-phase mass flow model [33] with optimized parameter set mainly for basal friction and entrainment coefficient, to simulate real-field landslides by back-calculating the Huascarán (Cordillera Blanca, Peru) events of 1962 and 1970. The results could reproduce the evolution of flow depths, velocities, travel times, and volumes of the events, indicating some challenges and uncertainties in simulating the complex mass flow events. Qiao et al. [35] also used r.avaflow 1 and the two-phase mass flow model 33 to simulate and analyze the run out characteristics of the catastrophic landslide that occurred in 2015 at Hongao construction waste dump-site in the Guangming New District of Shenzhen, China. They performed various model runs by varying solid density, solid volume fraction and the complex drag. 
This work mainly focuses on the simulation results, especially concerned with symmetry analyses of the flow in different generic slope topography with different rotational symmetries and differently shaped obstacles, the release areas, and also in different numerical discretization, employing the general two-phase mass flow model [33] through r.avaflow.

\section{Model Equations}

In the source codes of r.avaflow [1, different models from single to two-phase mass flows have been used. The general two-phase mass flow model of Pudasaini 33 has been further extended by including curvature effects in the mountain topography [10, erosion/deposition mechanics and related change in the basal topography, the ambient drag [22], and variable bed friction angles along with sophisticated constitutive models for the solid and fluid phases. The model [33] is a set of depth-integrated mass and momentum conservation equations for solid and fluid phase and in downslope and cross-slope directions both. However, in the numerical computation, the conversion of height into depth and vice-versa have been carried out in several steps.

In the two-phase model [33], the two phases are described by different material properties. The fluid phase is characterized by its material density $\rho_{f}$, viscosity $\eta_{f}$ and isotropic stress distribution. But the solid phase is characterized by its material density $\rho_{s}$, internal friction angle $\phi$, the basal friction angle $\delta$, an anisotropic stress distribution, and the lateral earth pressure coefficient, $K$. Here, the subscripts $s$ and $f$ are designated for the solid and the fluid phases respectively. Depth-averaged velocity components for fluid are $\mathbf{u}_{f}=\left(u_{f}, v_{f}\right)$ and those for solid are $\mathbf{u}_{s}=\left(u_{s}, v_{s}\right)$ in the down-slope $(x)$ and the cross-slope (y) directions, respectively. The flow depth is denoted by $h$, the solid-volume fraction by $\alpha_{s}$ and the fluid volume fraction by $\alpha_{f}=1-\alpha_{s}$. These field variables are computed as functions of space and time. The solid and fluid mass and momentum balance equations in the down-slope and the cross-slope directions are given by 33 .

$$
\begin{aligned}
& \frac{\partial}{\partial t}\left(\alpha_{s} h\right)+\frac{\partial}{\partial x}\left(\alpha_{s} h u_{s}\right)+\frac{\partial}{\partial y}\left(\alpha_{s} h v_{s}\right)=0 \\
& \frac{\partial}{\partial t}\left(\alpha_{f} h\right)+\frac{\partial}{\partial x}\left(\alpha_{f} h u_{f}\right)+\frac{\partial}{\partial y}\left(\alpha_{f} h v_{f}\right)=0 \\
& \frac{\partial}{\partial t}\left[\alpha_{s} h\left(u_{s}-\gamma \mathcal{C}\left(u_{f}-u_{s}\right)\right)\right]+\frac{\partial}{\partial x}\left[\alpha_{s} h\left(u_{s}^{2}-\gamma \mathcal{C}\left(u_{f}^{2}-u_{s}^{2}\right)+\frac{\beta_{x_{s}} h}{2}\right)\right] \\
& +\frac{\partial}{\partial y}\left[\alpha_{s} h\left(u_{s} v_{s}-\gamma \mathcal{C}\left(u_{f} v_{f}-u_{s} v_{s}\right)\right)\right]=h S_{x_{s}} \\
& \frac{\partial}{\partial t}\left[\alpha_{s} h\left(v_{s}-\gamma \mathcal{C}\left(v_{f}-v_{s}\right)\right)\right]+\frac{\partial}{\partial x}\left[\alpha_{s} h\left(u_{s} v_{s}-\gamma \mathcal{C}\left(u_{f} v_{f}-u_{s} v_{s}\right)\right)\right] \\
& +\frac{\partial}{\partial y}\left[\alpha_{s} h\left(v_{s}^{2}-\gamma \mathcal{C}\left(v_{f}^{2}-v_{s}^{2}\right)+\frac{\beta_{y_{s}} h}{2}\right)\right]=h S_{y_{s}} \\
& \frac{\partial}{\partial t}\left[\alpha_{f} h\left(u_{f}+\frac{\alpha_{s}}{\alpha_{f}} \mathcal{C}\left(u_{f}-u_{s}\right)\right)\right]+\frac{\partial}{\partial x}\left[\alpha_{f} h\left(u_{f}^{2}+\frac{\alpha_{s}}{\alpha_{f}} \mathcal{C}\left(u_{f}^{2}-u_{s}^{2}\right)+\frac{\beta_{x_{f}} h}{2}\right)\right] \\
& +\frac{\partial}{\partial y}\left[\alpha_{f} h\left(u_{f} v_{f}+\frac{\alpha_{s}}{\alpha_{f}} \mathcal{C}\left(u_{f} v_{f}-u_{s} v_{s}\right)\right)\right]=h S_{x_{f}} \\
& \frac{\partial}{\partial t}\left[\alpha_{f} h\left(v_{f}+\frac{\alpha_{s}}{\alpha_{f}} \mathcal{C}\left(v_{f}-v_{s}\right)\right)\right]+\frac{\partial}{\partial x}\left[\alpha_{f} h\left(u_{f} v_{f}+\frac{\alpha_{s}}{\alpha_{f}} \mathcal{C}\left(u_{f} v_{f}-u_{s} v_{s}\right)\right)\right]
\end{aligned}
$$




$$
+\frac{\partial}{\partial y}\left[\alpha_{f} h\left(v_{f}^{2}+\frac{\alpha_{s}}{\alpha_{f}} \mathcal{C}\left(v_{f}^{2}-v_{s}^{2}\right)+\frac{\beta_{y_{f}} h}{2}\right)\right]=h S_{y_{f}}
$$

where

$$
\beta_{x_{s}}=\varepsilon K_{x} p_{b_{s}}, \beta_{y_{s}}=\varepsilon K_{y} p_{b_{s}}, \beta_{x_{f}}=\beta_{y_{f}}=\varepsilon p_{b_{f}}, p_{b_{f}}=-g^{z}, p_{b_{s}}=(1-\gamma) p_{b_{f}} .
$$

The first two equations (1)-(2) are the depth averaged mass balances for solid and fluid phases respectively, and the last four equations are the depth averaged momentum balances for solid (3)-(4) and fluid (5)-(6), in $x$ - and $y$-directions respectively. The source terms in the right hand sides of the last four momentum equations are given by:

$$
\begin{aligned}
& S_{x_{s}}=\alpha_{s}\left[g^{x}-\frac{u_{s}}{\left|\mathbf{u}_{s}\right|} \tan \delta p_{b_{s}}-\varepsilon p_{b_{s}} \frac{\partial b}{\partial x}\right]-\varepsilon \alpha_{s} \gamma p_{b_{f}}\left[\frac{\partial h}{\partial x}+\frac{\partial b}{\partial x}\right] \\
& +C_{D G}\left(u_{f}-u_{s}\right)\left|\mathbf{u}_{f}-\mathbf{u}_{s}\right|^{J-1}, \\
& S_{y_{s}}=\alpha_{s}\left[g^{y}-\frac{v_{s}}{\left|\mathbf{u}_{s}\right|} \tan \delta p_{b_{s}}-\varepsilon p_{b_{s}} \frac{\partial b}{\partial y}\right]-\varepsilon \alpha_{s} \gamma p_{b_{f}}\left[\frac{\partial h}{\partial y}+\frac{\partial b}{\partial y}\right] \\
& +C_{D G}\left(v_{f}-v_{s}\right)\left|\mathbf{u}_{f}-\mathbf{u}_{s}\right|^{J-1} \\
& S_{x_{f}}=\alpha_{f}\left[g^{x}-\varepsilon\left[\frac{1}{2} p_{b_{f}} \frac{h}{\alpha_{f}} \frac{\partial \alpha_{s}}{\partial x}+p_{b_{f}} \frac{\partial b}{\partial x}-\frac{1}{\alpha_{f} N_{R}}\left\{2 \frac{\partial^{2} u_{f}}{\partial x^{2}}+\frac{\partial^{2} v_{f}}{\partial y \partial x}+\frac{\partial^{2} u_{f}}{\partial y^{2}}-\frac{\chi u_{f}}{\varepsilon^{2} h^{2}}\right\}\right.\right. \\
& +\frac{1}{\alpha_{f} N_{R_{\mathcal{A}}}}\left\{2 \frac{\partial}{\partial x}\left(\frac{\partial \alpha_{s}}{\partial x}\left(u_{f}-u_{s}\right)\right)+\frac{\partial}{\partial y}\left(\frac{\partial \alpha_{s}}{\partial x}\left(v_{f}-v_{s}\right)+\frac{\partial \alpha_{s}}{\partial y}\left(u_{f}-u_{s}\right)\right)\right\} \\
& \left.\left.-\frac{\xi \alpha_{s}\left(u_{f}-u_{s}\right)}{\varepsilon^{2} \alpha_{f} N_{R_{\mathcal{A}}} h^{2}}\right]\right]-\frac{1}{\gamma} C_{D G}\left(u_{f}-u_{s}\right)\left|\mathbf{u}_{f}-\mathbf{u}_{s}\right|^{J-1} \\
& S_{y_{f}}=\alpha_{f}\left[g^{y}-\varepsilon\left[\frac{1}{2} p_{b_{f}} \frac{h}{\alpha_{f}} \frac{\partial \alpha_{s}}{\partial y}+p_{b_{f}} \frac{\partial b}{\partial y}-\frac{1}{\alpha_{f} N_{R}}\left\{2 \frac{\partial^{2} v_{f}}{\partial y^{2}}+\frac{\partial^{2} u_{f}}{\partial x \partial y}+\frac{\partial^{2} v_{f}}{\partial x^{2}}-\frac{\chi v_{f}}{\varepsilon^{2} h^{2}}\right\}\right.\right. \\
& +\frac{1}{\alpha_{f} N_{R_{\mathcal{A}}}}\left\{2 \frac{\partial}{\partial y}\left(\frac{\partial \alpha_{s}}{\partial y}\left(v_{f}-v_{s}\right)\right)+\frac{\partial}{\partial x}\left(\frac{\partial \alpha_{s}}{\partial y}\left(u_{f}-u_{s}\right)+\frac{\partial \alpha_{s}}{\partial x}\left(v_{f}-v_{s}\right)\right)\right\} \\
& \left.\left.-\frac{\xi \alpha_{s}\left(v_{f}-v_{s}\right)}{\varepsilon^{2} \alpha_{f} N_{R_{\mathcal{A}}} h^{2}}\right]\right]-\frac{1}{\gamma} C_{D G}\left(v_{f}-v_{s}\right)\left|\mathbf{u}_{f}-\mathbf{u}_{s}\right|^{J-1},
\end{aligned}
$$

where

$$
\begin{gathered}
C_{D G}=\frac{\alpha_{s} \alpha_{f}(1-\gamma)}{\left[\varepsilon \mathcal{U}_{T}\left\{\mathcal{P} \mathcal{F}\left(R e_{p}\right)+(1-\mathcal{P}) \mathcal{G}\left(R e_{p}\right)\right\}\right]^{J}}, \quad \mathcal{F}=\frac{\gamma}{180}\left(\frac{\alpha_{f}}{\alpha_{s}}\right)^{3} R e_{p}, \\
\gamma=\frac{\rho_{f}}{\rho_{s}}, \quad \mathcal{G}=\alpha_{f}^{M\left(R e_{p}\right)-1}, \quad R e_{p}=\frac{\rho_{f} d \mathcal{U}_{T}}{\eta_{f}}, \quad N_{R}=\frac{\sqrt{g L} H \rho_{f}}{\alpha_{f} \eta_{f}}, \quad N_{R_{\mathcal{A}}}=\frac{\sqrt{g L} H \rho_{f}}{\mathcal{A} \eta_{f}} .
\end{gathered}
$$


In the above equations, $x, y$ and $z$ are usual spatial coordinates, $t$ is time, and $g^{x}, g^{y}$ and $g^{z}$ are the respective components of gravitational acceleration. $H$ and $L$ are the typical depth and the length of the flow, $\varepsilon=H / L$ is their aspect ratio. $\mu=\tan \delta$ is the basal friction coefficient with $\delta$ as the basal friction angle. $K$ is the earth pressure coefficient, and $C_{D G}$ is the generalized drag coefficient. The generalized drag is modeled by a linear combination of fluid-like $\operatorname{drag}(\mathcal{F})$ and solid-like $\operatorname{drag}(\mathcal{G}) . J=1$ or 2 respectively associate simple linear (laminar-type, at low velocity), or quadratic (turbulent-type, at high velocity) drag. $\mathcal{U}_{T}$ is the terminal velocity of a particle and $\mathcal{P} \in[0,1]$ is an interpolation parameter that combines the solidlike $(\mathcal{G})$ and fluid-like $(\mathcal{F})$ drag contributions to flow resistance. $\gamma$ is the density ratio of the fluid and solid, $\mathcal{C}$ is the virtual mass coefficient, $\eta_{f}$ is the fluid viscosity, $M$ is a function of the particle Reynolds number $\left(R e_{p}\right), \chi$ includes vertical shearing of fluid velocity, and $\xi$ takes into account different distributions of $\alpha_{s}$. $\mathcal{A}$ is the mobility of the fluid at the interface, and $N_{R}$ and $N_{R_{\mathcal{A}}}$ respectively are the quasi-Reynolds number and mobility-Reynolds number associated with the classical Newtonian and enhanced non-Newtonian fluid viscous stresses. Slope topography is given by $b=b(x, y)$.

The above six equations $([1]-(6))$ can be written in a compact vectorial form, as

$$
\frac{\partial \mathbf{T}(\mathbf{w})}{\partial t}+\frac{\partial \mathbf{f}(\mathbf{w})}{\partial x}+\frac{\partial \mathbf{g}(\mathbf{w})}{\partial y}=\mathbf{s}(\mathbf{w})
$$

where $\mathbf{w}$ denotes the vector of conservative variables $h_{s}, h_{f}, m_{x_{s}}\left(=h_{s} u_{s}\right), m_{x_{f}}\left(=h_{f} u_{f}\right), m_{y_{s}}\left(=h_{s} v_{s}\right)$ and $m_{y_{f}}\left(=h_{f} v_{f}\right)$. Also, $\mathbf{f}$ and $\mathbf{g}$ are the transport fluxes in the $x$ - and $y$-directions, respectively, and $\mathbf{s}$ is the source term, i.e.,

$$
\begin{aligned}
& \mathbf{T}(\mathbf{w})=\left(\begin{array}{c}
h_{s} \\
h_{f} \\
m_{x_{s}}-\gamma \mathcal{C}\left(m_{x_{f}} h_{s} / h_{f}-m_{x_{s}}\right) \\
m_{y_{s}}-\gamma \mathcal{C}\left(m_{y_{f}} h_{s} / h_{f}-m_{y_{s}}\right) \\
m_{x_{f}}+\mathcal{C}\left(m_{x_{f}} h_{s} / h_{f}-m_{x_{s}}\right) \\
m_{y_{f}}+\mathcal{C}\left(m_{y_{f}} h_{s} / h_{f}-m_{y_{f}}\right)
\end{array}\right), \quad \mathbf{w}=\left(\begin{array}{c}
h_{s} \\
h_{f} \\
m_{x_{s}} \\
m_{y_{s}} \\
m_{x_{f}} \\
m_{y_{f}}
\end{array}\right) \\
& \mathbf{f}=\left(\begin{array}{c}
m_{x_{s}} \\
m_{x_{f}} \\
m_{x_{s}}^{2} / h_{s}-\gamma \mathcal{C}\left(m_{x_{f}}^{2} h_{s} / h_{f}^{2}-m_{x_{s}}^{2} / h_{s}\right)+\beta_{x_{s}} h_{s} h / 2 \\
m_{x_{s}} m_{y_{s}} / h_{s}-\gamma \mathcal{C}\left(m_{x_{f}} m_{y_{f}} h_{s} / h_{f}^{2}-m_{x_{s}} m_{y_{s}} / h_{s}\right) \\
m_{x_{f}}^{2} / h_{f}+\mathcal{C}\left(m_{x_{f}}^{2} h_{s} / h_{f}^{2}-m_{x_{s}}^{2} / h_{s}\right)+\beta_{x_{f}} h_{f} h / 2 \\
m_{x_{f}} m_{y_{f}} / h_{f}+\mathcal{C}\left(m_{x_{f}} m_{y_{f}} h_{s} / h_{f}^{2}-m_{x_{s}} m_{y_{s}} / h_{s}\right)
\end{array}\right) \\
& \mathbf{g}=\left(\begin{array}{c}
m_{y_{s}} \\
m_{y_{f}} \\
m_{x_{s}} m_{y_{s}} / h_{s}-\gamma \mathcal{C}\left(m_{x_{f}} m_{y_{f}} h_{s} / h_{f}^{2}-m_{x_{s}} m_{y_{s}} / h_{s}\right) \\
m_{y_{s}}^{2} / h_{s}-\gamma \mathcal{C}\left(m_{x_{f}}^{2} h_{s} / h_{f}^{2}-m_{y_{s}}^{2} / h_{s}\right)+\beta_{y_{s}} h_{s} h / 2 \\
m_{x_{f}} m_{y_{f}} / h_{f}+\mathcal{C}\left(m_{x_{f}} m_{y_{f}} h_{s} / h_{f}^{2}-m_{x_{s}} m_{y_{s}} / h_{f}\right) \\
m_{x_{f}}^{2} / h_{f}+\mathcal{C}\left(m_{y_{f}}^{2} h_{s} / h_{f}^{2}-m_{y_{s}}^{2} / h_{s}\right)+\beta_{y_{f}} h_{f} h / 2
\end{array}\right), \quad \mathbf{s}=\left(\begin{array}{c}
0 \\
0 \\
h S_{x_{s}} \\
h S_{y_{s}} \\
h S_{x_{f}} \\
h S_{y_{f}}
\end{array}\right)
\end{aligned}
$$

where these expressions are computed in the conservative variables. In the general two-phase debris flow model proposed by Pudasaini [33, the phase-averaged balance equations for mass and momentum conservations is considered and the following assumptions are made: surface tension is negligible, interfacial solid and fluid pressures are identical to the (true) fluid pressure, the solid and fluid components are incompressible (the true densities are constants), and no phase change occurs [17, 21, 33, 34. 
Simulations for Flow-Symmetry Through r.avaflow in the General Two-Phase Mass Flow Model

\section{Numerical Methods and Choice of Parameters}

Total Variation Diminishing (TVD) Non-Oscillatory Central (NOC) scheme has been implemented to numerically integrate the model (12). TVD-NOC scheme are effective even when the shocks are produced due to rapid motion and the large deformation during the flow and obstacle-interactions [15, 24, 29, 32, 39. Two-dimensional non-oscillatory central differencing (NOC) scheme was previously used for numerical simulation of granular avalanche flows 32. It is a predictor-corrector method that involves the following two steps. (i) The grid values are first predicted according to the non-oscillatory reconstructions from the given cell averages. (ii) A staggered averaging is introduced, together with the predicted mid-values as the second corrector step, to determine the full evolution of these averages. By this, a high-order, non-oscillatory central scheme results in. For spatial and temporal discretization of the domain and the discretization of the model equations, one can refer to Pudasaini and Hutter 32 .

Different generic topographies and initial debris masses are created by using MATLAB and the data are imported as raster and release using GRASS as ascii files. r.avaflow employs the main computational code in C-language and gives the numerical outputs. At the end of each model run, the visualization tool enables to output the profiles of the change in basal topography, depth, solid and fluid velocities, pressure and kinetic energy along with their maximum stage [1]. In most of the simulations carried out here, we kept the tracking of edges and conservation of volumes always activated. The common parameters chosen for simulations are $\phi=40^{\circ}, \delta=25^{\circ}, \rho_{f}=1,000 \mathrm{~kg} \mathrm{~m}^{-3}, \rho_{s}=2,700 \mathrm{~kg} \mathrm{~m}^{-3}, \log \left(N_{R}\right)=4.477$, $\log \left(N_{R_{\mathcal{A}}}\right)=3.0, \mathcal{U}_{T}=1.0 \mathrm{~m} \mathrm{~s}^{-1}, \mathcal{P}=0.5, J=1$ (only linear drag), $\mathcal{C}=0.5$. The initial release mass, the employed material parameters and the slope topography are the initial conditions. As boundary condition, the upper free surface is traction free, and Coulomb sliding for solid and no-slip for fluid are employed at the flow-base. In case of the simulations with obstacles, the obstacle geometry locally changes the topographic gradient of the slope. The obstacle-induced spatial derivatives $\partial b / \partial x$ and $\partial b / \partial y$ enter the model equations to account for the flow-obstacle-interactions [20, 23, 24] The parameters and other initial conditions are saved in a script file. The 20160706 (July 7, 2016) version has been mostly used for the simulation results presented here [1].

\section{Simulation Results Through r.avaflow}

Among many factors that ensures a robust computational tool, the very important one is that the flow must be symmetric in the normal situation. Asymmetry may arise if the raster and computational resolution are not properly addressed. To investigate into the asymmetric issues, another idea is to check whether the computation technique is direction invariant or not. In real flow situation, it is equivalent that whether the identical release and topography will produce same flow scenario in the north west, south east or any other flanks of the mountains. In the simulations, we could have rotated the flow plane and the release mass in the desired angle. Another equally wise idea is to consider the topography with different rotational symmetries, where identical release masses are triggered at once in the identical flanks all around and observe the flow scenario. In all the following simulation figures, we have only presented the three panels. The left is for maximum flow height, the middle for the final change in basal topography and the right for maximum flow kinetic energy.

\subsection{Rotational Symmetry on Different Topographies}

To check the asymmetries in the flow during simulation, we considered different topographies with different rotational symmetry where identical release masses slide down together. For Fig. 17Fig. 3 , the $2 \mathrm{D}$-computational domain is $[-4000,4000] \times[-4000,4000]$ in metre and centre at the origin. For Fig. 1 Fig. 3, the raster resolution and the computational resolution are both of $10 \mathrm{~m}$ each. The release is a hemispherical mass of radius $50 \mathrm{~m}$ constituting a homogeneous mixture of $80 \%$ solid and $20 \%$ fluid. The height of each of the differently-based pyramids is $1000 \mathrm{~m}$.

(i) Triangle-based pyramid as topography with hemispherical releases

As the topography, we consider a triangle-based pyramid, or a tetrahedron with rotational symmetry 

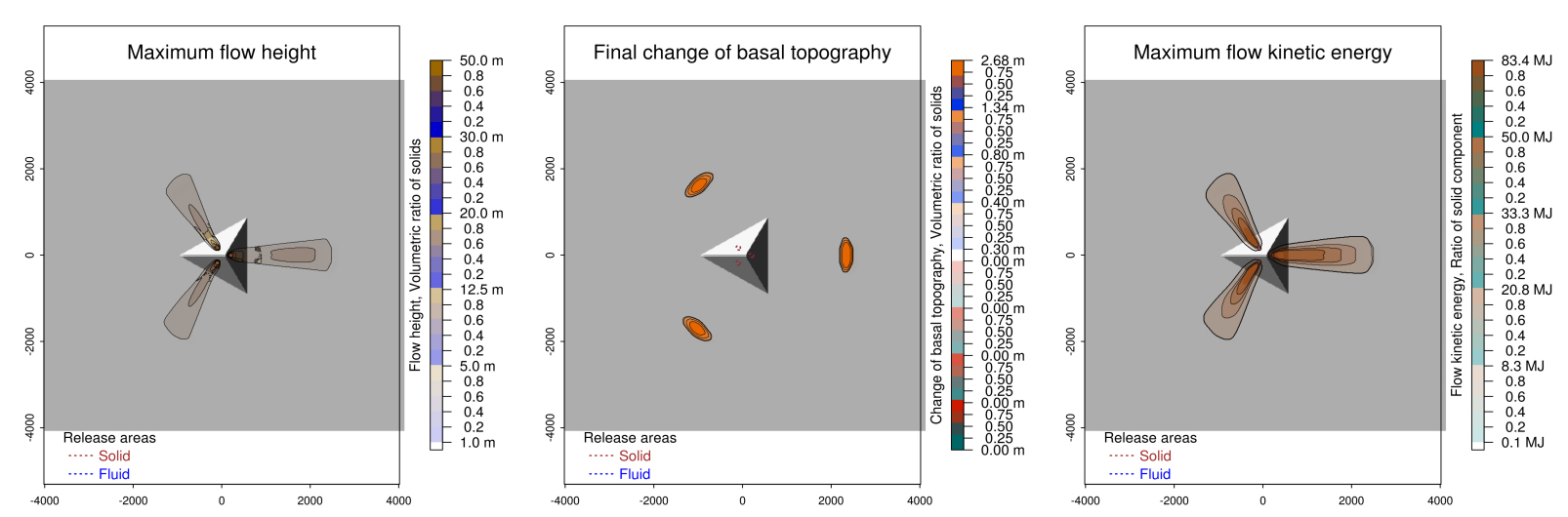

Figure 1: Topography is a plane with equilateral triangular based pyramid at the centre. The vertices of the triangular base are at $(-1000,0),(500,-500 \sqrt{ } 3)$ and $(500,500 \sqrt{ } 3)$.

$=3$. In all three lateral faces of the tetrahedron, identical release mass centred at the corresponding height of $(-100 \sqrt{ } 2,-100 \sqrt{ } 3),(-100 \sqrt{ } 2,100 \sqrt{ } 3)$ and $(200,0)$ are kept. The slope angle of each face is $\cos ^{-1}\left(\frac{1}{\sqrt{5}}\right) \approx 63.43^{\circ}$. At a first glance, the flow seems to be symmetrical but it is not perfect. The flow from the right hand face looks a bit different from the other two in a very close scrutiny. It seems to be more dispersed and traveled longer. The final depositions of the solid phase are in the form of a semi-ellipsoid with height of $2.88 \mathrm{~m}$. The profiles for maximum flow kinetic energy form layered structures, which show maxima (up to 83.4 Mega Joule) along the flow direction, which go on decreasing cross wise in the dispersed regions. The employed MATLAB codes for the raster and release are presented in Appendix-A.

\section{(ii) Square based pyramid as topography with hemispherical releases}

Next, we present the case with four rotational symmetries in the topography by considering the four faces of a square-based pyramid mounted at the centre of the plane. Each face has the slope angle of $45^{\circ}$. In this case, the results look very much improved (See Fig. 20 in the sense of symmetry, as compared to the previous case (Fig. 1). Due to the decreased slope angle, the run out distance is shorter in comparison to the previous case (Fig. 1), so that the maximum heights of the depositions are increased to $3.52 \mathrm{~m}$ in this case. The decreased slope angle also results in the decreased velocity to give decreased maximum flow kinetic energy (a maximum of 54.3 Mega Joule). The codes for this simulation are presented in Appendix-B.
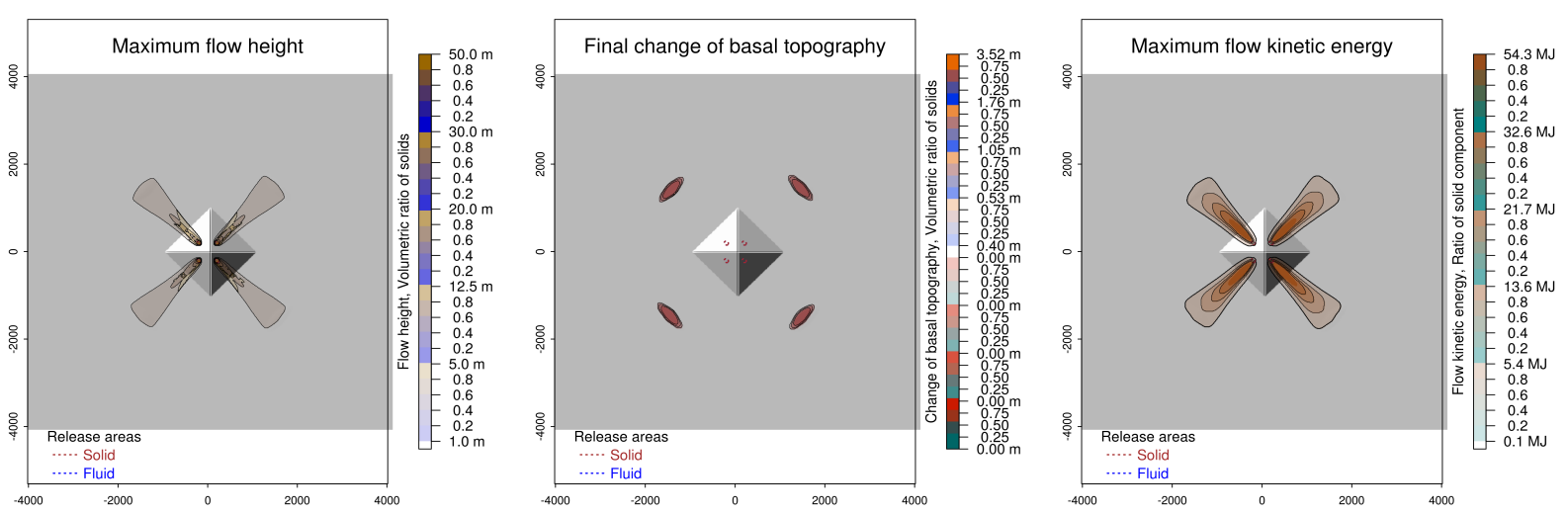

Figure 2: Topography is a plane with a square based pyramid at the centre of the plane with vertices of the base at $(-1000,0),(0,1000),(0,-1000)$ and $(1000,0)$. The centre of the four release masses are at the corresponding points on the faces of the points $( \pm 100,0)$ and $(0, \pm 100)$. 

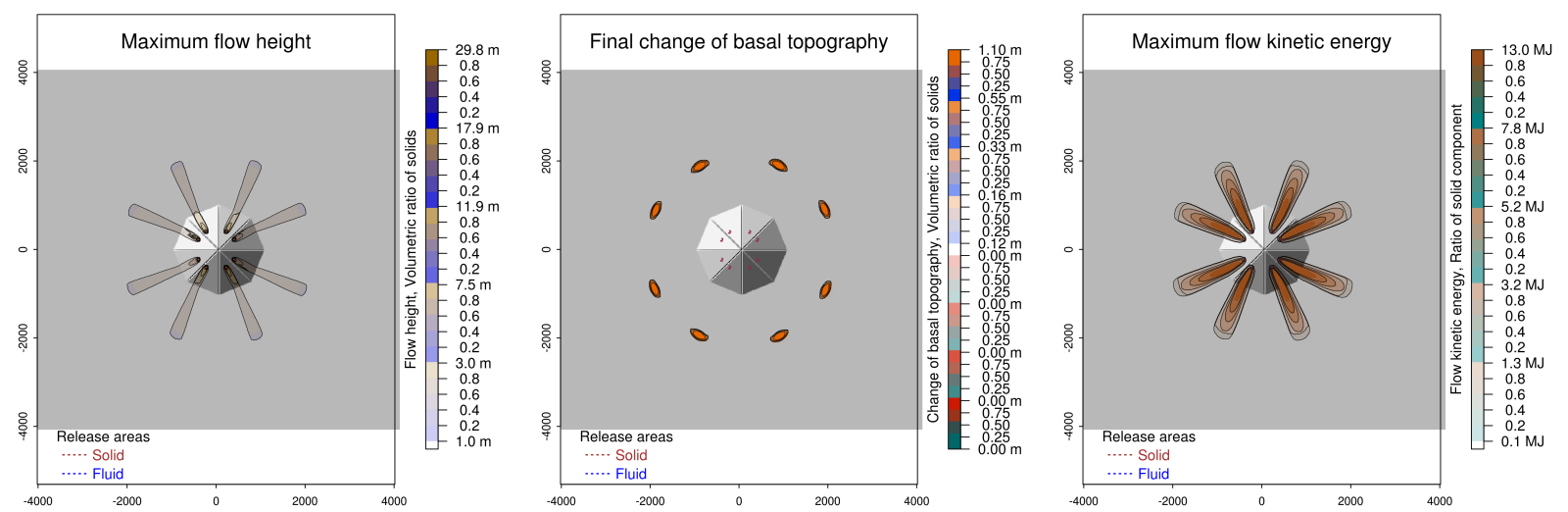

Figure 3: Octagon-based pyramid at the centre of the plane. The apex is at $(0,0,1000)$. The vertices of the base are at $( \pm r, 0),(0, \pm r),( \pm r p, \pm r p)$ where $r=2000 \mathrm{~m}$ and $p=\frac{1}{\sqrt{ } 2}$. The release masses have the centers at the corresponding points on the faces at $( \pm 400, \pm 200),( \pm 200, \pm 400)$. Thus, the slope angle of each face is $\cos ^{-1}\left(\frac{1}{\sqrt{8-2 \sqrt{2}}}\right) \approx 28.42^{\circ}$

\section{(iii) Octagon-based pyramid as topography with hemispherical releases}

Now, we have the octagon-based pyramid placed at the centre of the plane as topography (See Fig. 3) so that the rotational symmetry has been increased to eight. The identical release mass at the same height in all the eight faces start flowing at the same time. We can clearly see that all the eight flows have almost the same run out extents. The results seem to be very much improved with respect to the symmetry. To check the symmetry in even better way, we have drawn a profile line in yellow that passes through the centre and mid-way of two opposite flanks among the eight. The two respective flows seem to be almost bilaterally symmetrical about this line. Due to the sharp decrease in the slope angle, the velocity of the flow is decreased, that results in the remarkable decrease in the flow kinetic energy of the flow. The employed codes for the octagonal pyramid and the release masses in all the eight faces have been presented in Appendix-C.

It is necessary to check the possible causes of asymmetries in the results. The vertices and edges of the pyramids do not have all the rational coordinates. As the resolution of the computation is of $10 \mathrm{~m}$, the computational nodes cannot take into account of all the vertices and edges of the pyramids symmetrically. Another possible cause may be that the depth-integrated model is suitable only when the release height is substantially smaller than the extent of the mass flows.

Next, we check the symmetry for some flows past obstacles.

\subsection{Flow Past Obstacles}

Here, we consider the symmetrical release mass (a hemispherical release) and symmetrical obstacles (triaglebased and square-based pyramidal structures) with respect to the downslope and the cross-slope directions.

\section{(i) Flow past a triangle-based pyramid as an obstacle}

In this case, a hemispherical release of radius $50 \mathrm{~m}$ centred at $(200,0) \mathrm{m}$ slides down an inclined plane inclined at $45^{\circ}$ up to $1000 \mathrm{~m}$ with the ground moves further in the run out zone. The flow suddenly encounters the forward-facing triangle-based pyramid (tetrahedron) of height $200 \mathrm{~m}$. The two sharp edges of the tetrahedron deflect the flowing mass into two streams and the mass gets deposited into two lobes. The deposited mass is in the form of two cross wise elongated lobes that merges near the central line $(y=0)$. As the deposited lobes are elongated and dispersed, the maximum depth of the deposition is only 

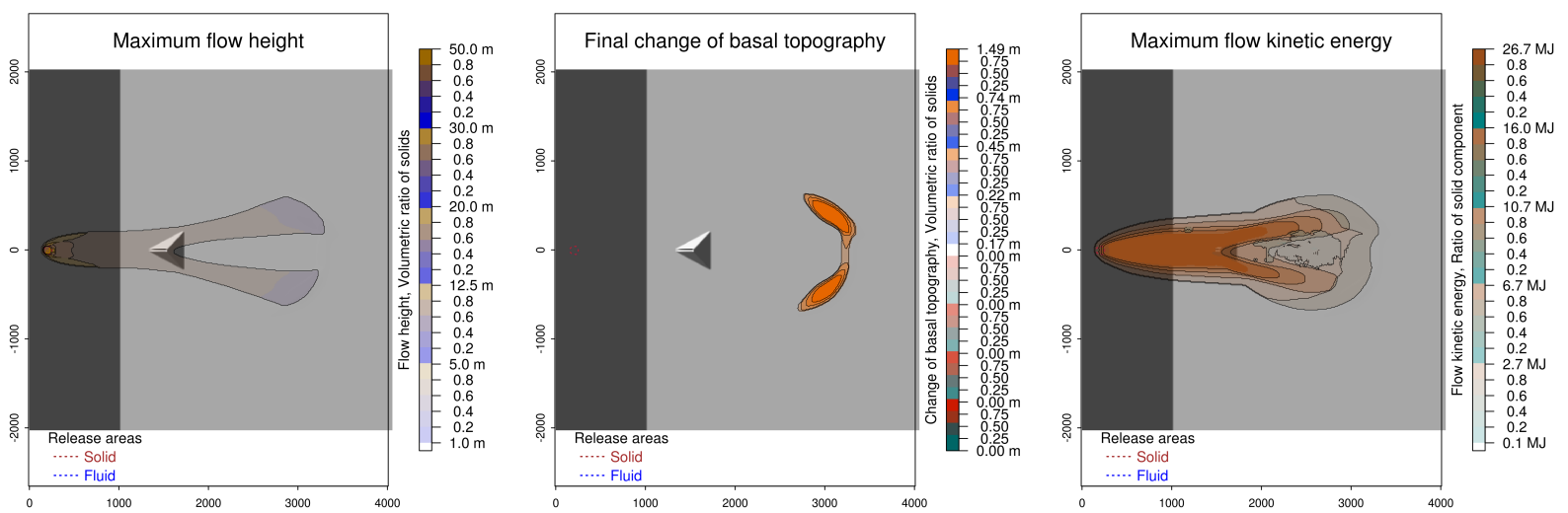

Figure 4: Flow past a triangular based pyramid as an obstacle when a hemispherical release of radius $50 \mathrm{~m}$ and centred at $(200,0)$ slides down an inclined plane inclined at $45^{\circ}$ with the ground moves further in the run out zone after $1000 \mathrm{~m}$ suddenly encounters the pyramid of height $200 \mathrm{~m}$. The apex is at $(1500,0,200)$ and the vertices of the bases are at $(1300,0,0),(1700,295,0)$ and $(1700,-295,0)$.

of $1.49 \mathrm{~m}$. The front of the flow reaches at the downslope distance of $3000 \mathrm{~m}$. The deposited lobes are different in shapes. There is a considerable asymmetry in the deposited lobes, in the middle panel of Fig. 4. Much more asymmetries are seen on the kinetic energy profile (Fig. 4. Right). The maximum flow kinetic energy is of 26.7 MJ that occurs in the main flow lines. The flow kinetic energy decreases along the lateral sides of the flow.

\section{(ii) Flow past a square-based pyramid as an obstacle}

In Fig. 5, the flow scenario and the deflection are presented where the obstacle is in the form of a squarebased pyramid. As the impacting faces have lower deflection angle than Fig. 4, the mass has not been deflected substantially in comparison to Fig. 4 and the mass is found to be deposited in a dumbbell shape at around $3000 \mathrm{~m}$. The symmetry is little more improved than in Fig. 4. In these obstacle interactions also, we found a lot of asymmetries. Asymmetries increased along with the increased size of the obstacles. In case of square based pyramid, there is less asymmetry. Thus, more the edges and vertices have irrational points, more is the asymmetry. So, we guess that the asymmetry is mainly due to the raster and computational resolutions, not in harmony with the geometry of the raster and obstacles.
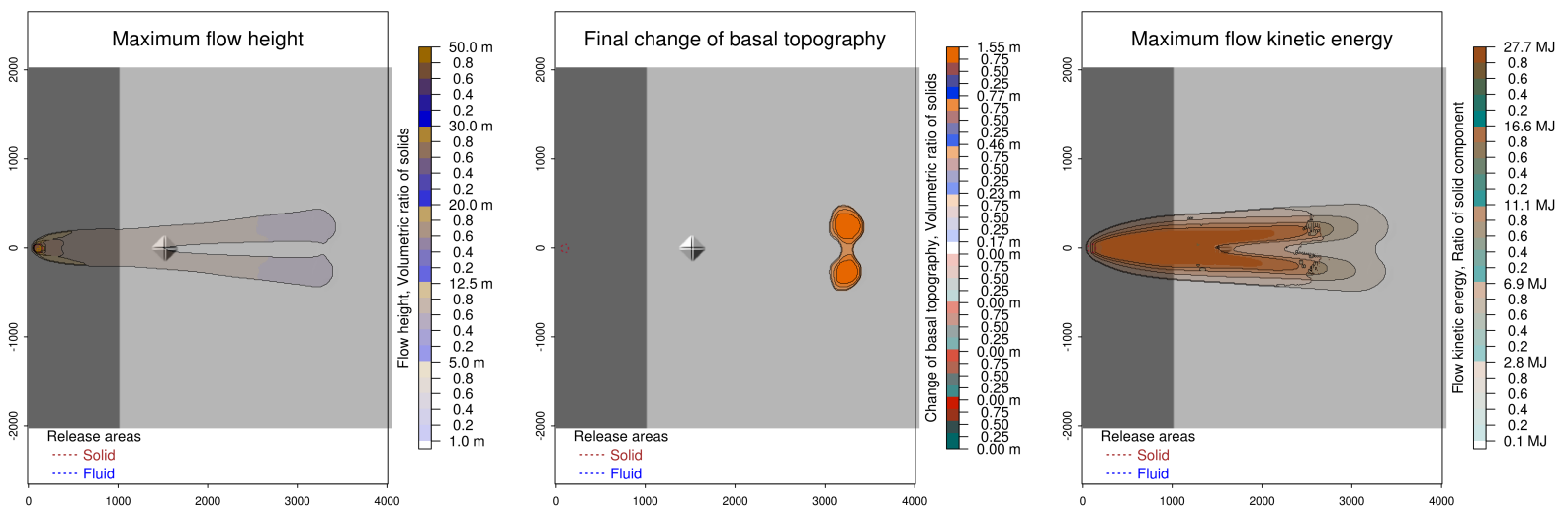

Figure 5: Flow past a square based pyramid as an obstacle when a hemispherical release of radius $50 \mathrm{~m}$ and centred at $(200,0)$ slides down an inclined plane inclined at $45^{\circ}$ with the ground moves further in the run out zone after $1000 \mathrm{~m}$ suddenly encounters the pyramid of height $200 \mathrm{~m}$. The apex is at $(1500,0,200)$ and the vertices of the bases are at $(1350,0,0),(1500, \pm 150,0)$ and $(1650,0,0)$. 
Simulations for Flow-Symmetry Through r.avaflow in the General Two-Phase Mass Flow Model

\section{Discussion and Summary}

Here we focused on the symmetric issues in the flow in different rotational symmetries and the obstacle shapes employing the general two-phase mass flow model [33] and the open source computational codes r.avaflow. While performing many simulation through r.avaflow, we found that the tool r.avaflow is sophisticated and has many user friendly facilities like

- executing the outputs as the numerical and geometrical plots of the entire flow scenario (change of basal topography, flow depths, velocities, pressures and kinetic energy).

- giving information about the problems arouse like numerical failures, overflow, stopping and many other error massage on time.

- can be used for large scale geophysical events, too.

- suitable for different mass flow models, from single to two-phase.

- not fit for small scaled flows.

- activating or deactivating different modes like tracking of edges, stopping, conversion of volumes, entrainment and deposition etc. by means of function control.

However, some of the problems aroused, which are also listed below

- numerical failures in finer resolutions for some topographies and steep slopes.

- volume loss also occurred in each time step although the conservation of volume.

- the version (20160818) is found to be more prone to numerical failures.

Symmetricity of the flow is more improved for the increased number of rotational symmetry in the slope topography. In case of obstacle interactions, the asymmetry also aroused. Another important issue is of the staggered grids. In case of entrainment and deposition, the staggered grids may not be appropriate. However, Non Oscillatory Central (NOC) scheme always demands the proper use of staggered grids, which is yet to be managed well in the numerics.

Acknowledgments: This work is a part of our research stay for three months at Department of Natural Hazards, Austrian Research Center for Forests (BFW), Innsbruck, Austria, to work for r.avaflow Project from July to September, 2016, supported by the transnational research project, "Development of a GIS-based Open Source Simulation Tool for modeling General Avalanche and Debris Flows over Natural Topography", research project PU 386/3-1 (DFG: German Research Foundation); I 1600-N30 (FWF: Austrian Science Fund). We gratefully acknowledge to

- Dr. Jan-Thomas Fischer, Federal Research and Training Center for Forests, Natural Hazards - BFW, Department of Natural Hazards, Rennweg 1, 6020 Innsbruck, Austria.

- Martin Mergili, Institute of Applied Geology, University of Natural Resources and Life Science (BOKU), Vienna, Austria.

- Shiva P. Pudasaini, Institute of Geosciences and Meteorology, Geophysics Section, University of Bonn, Germany.

for their guidance for the work.

\section{Appendix}

A. MATLAB Codes for Fig. 1 for the raster and release are given below: 


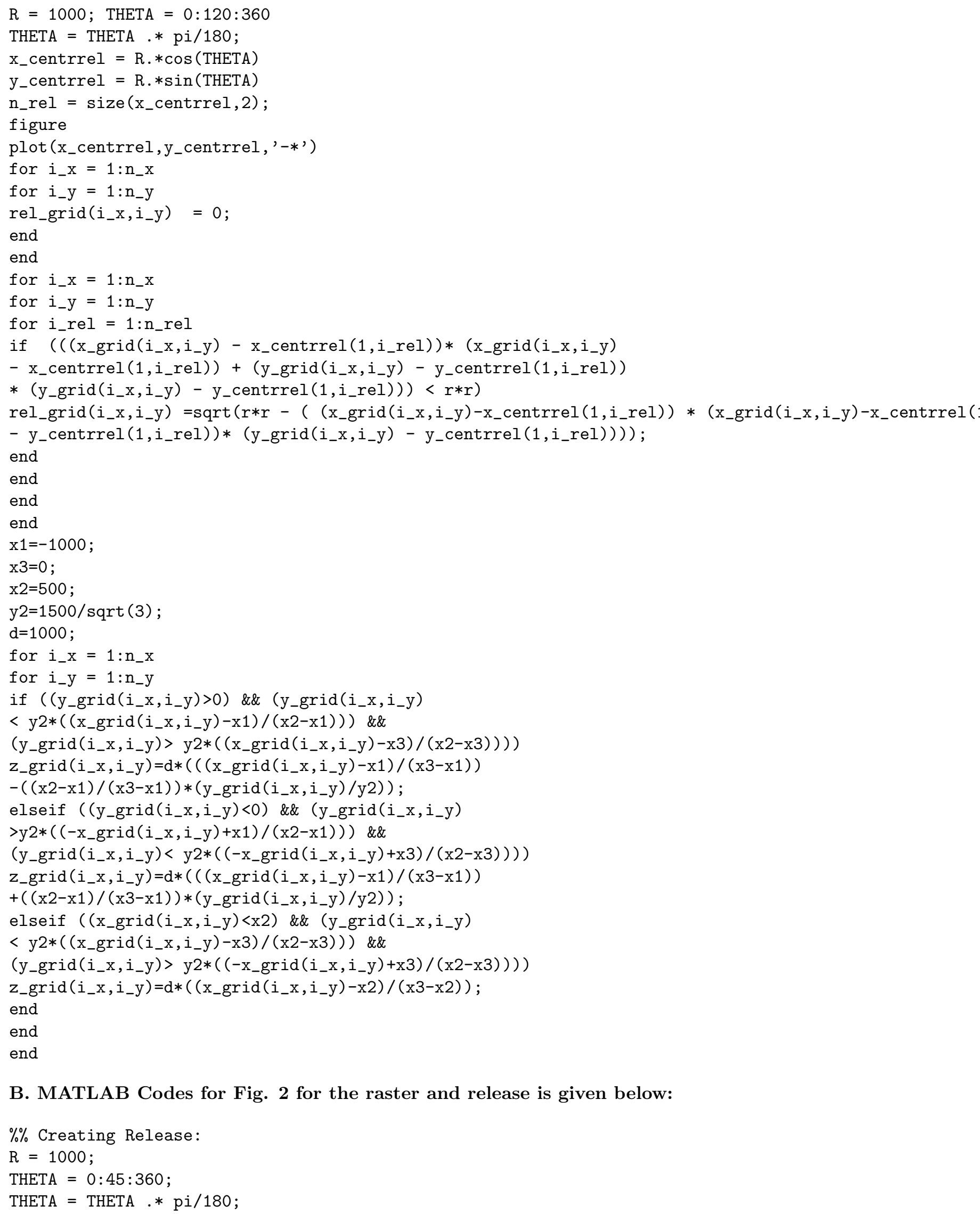

B. MATLAB Codes for Fig. 2 for the raster and release is given below:

$\%$ Creating Release:

$\mathrm{R}=1000$;

THETA $=0: 45: 360$;

THETA $=$ THETA.$*$ pi $/ 180 ;$ 


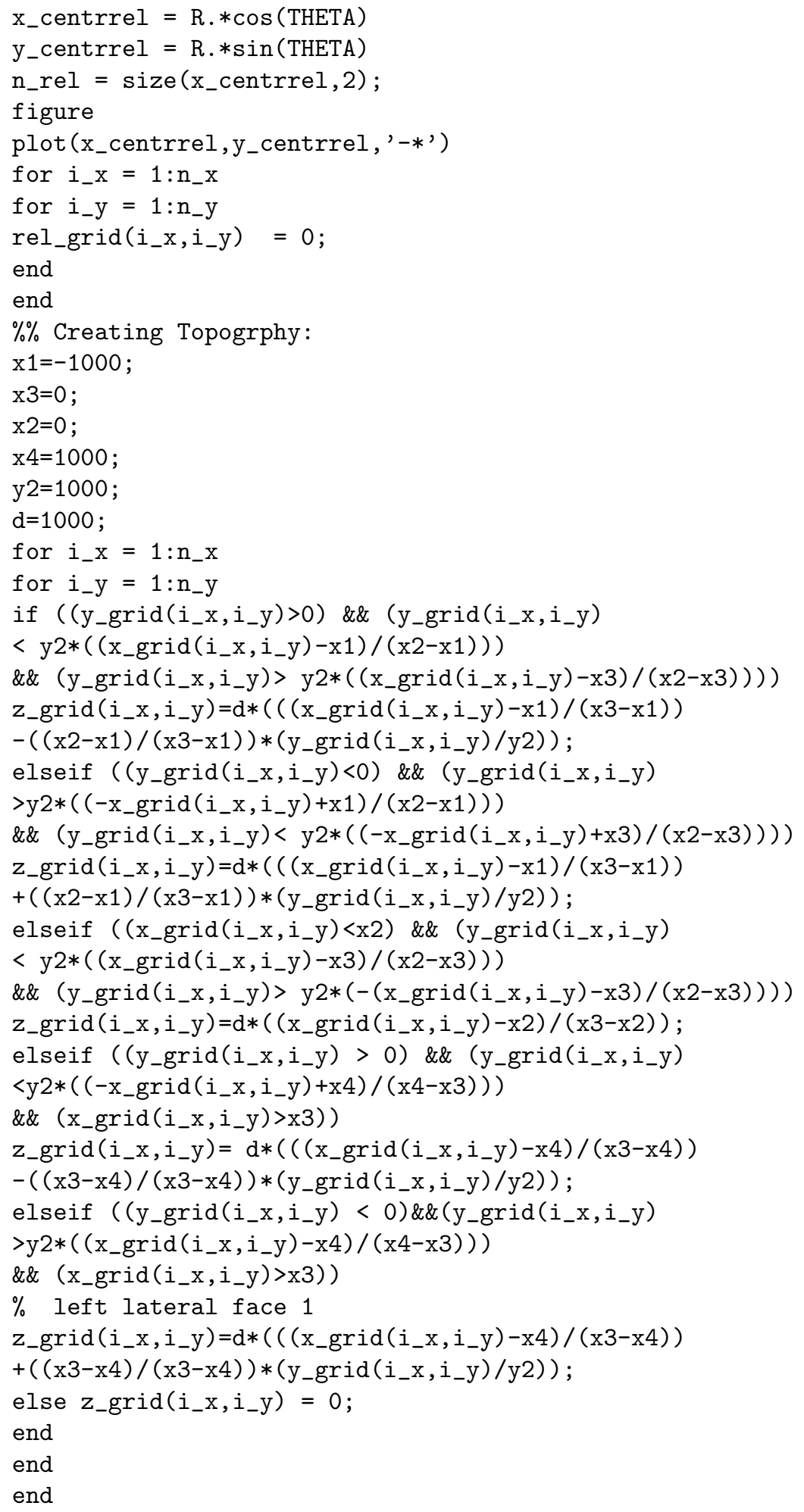

C. MATLAB Codes for Fig. 3 for the octagonal pyramid and the release masses in all the eight faces are given below.

$\%$ Creating Release:

$\mathrm{R}=1000$;

THETA $=45 / 2: 45: 360+45 / 2$ 


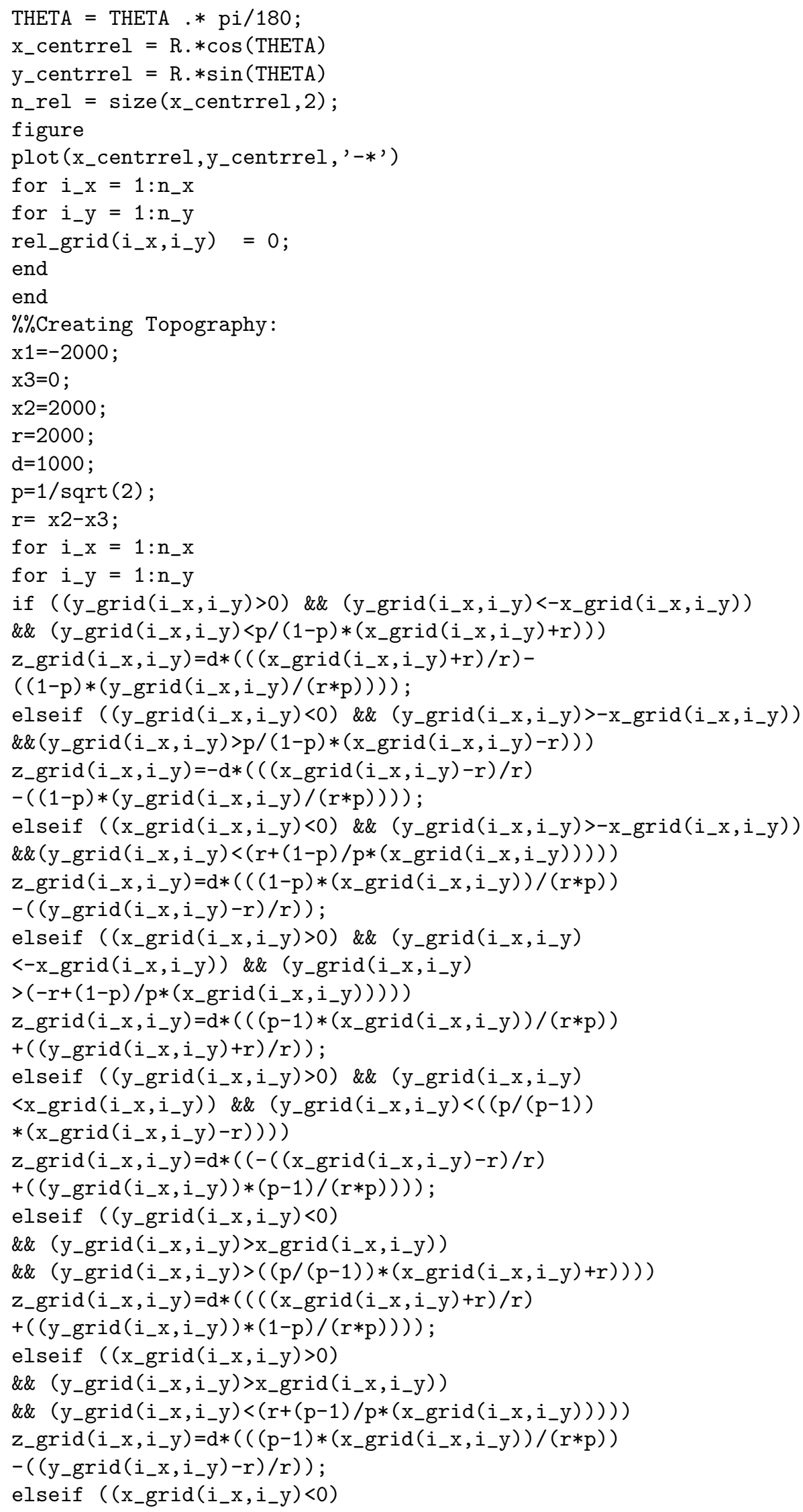




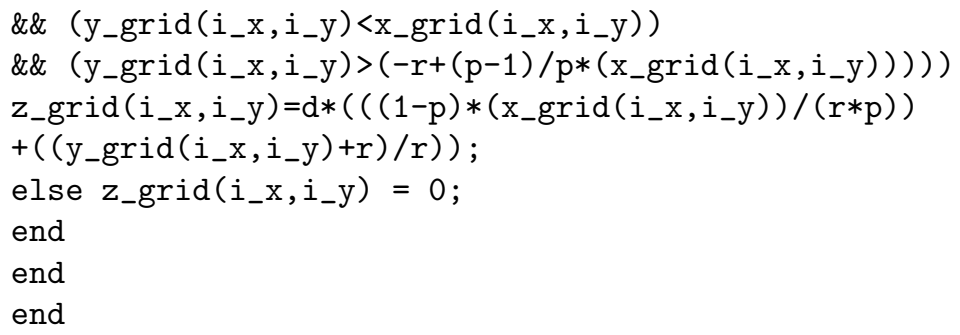

\section{References}

[1] www.avaflow.org

[2] www.openfoam.com

[3] Bagnold RA (1954) Experiments on a gravity-free dispersion of large solid spheres in a Newtonian fluid under shear. Proc. R. Soc. Lond., Ser. A, 225, 49-63

[4] Cageo P (2014) Fluid-Particle Interaction in Geophysical Flows: Debris Flow PhD Thesis, University of Nottingham, England

[5] Chen CL (1988) Generalized viscoplastic modelling of debris flow. J. Hydraul. Res., 114(3), 237-258

[6] Cui X and Gray J (2013) Gravity driven granular free-surface flow around a circular cylinder. J. Fluid Mech., 720, 314-337

[7] Cui P, Zeng C and Lei Yu (2015) Experimental analysis on the impact force of viscous debris flow. Earth Surf. Process. Landforms, 40, 1644-1655 (doi: 10.1002/esp.3744)

[8] Douglas S (2016) Numerical Modeling of Extreme Hydrodynamic Loading and Pneumatic Long Wave Generation: Application of a Multiphase Fluid Model. PhD Dissertation, University of Ottawa, Canada

[9] Fernandez-Nieto ED, Bouchut F, Bresch D, Castro Daz MJ and Mangeney A (2008) A new SavageHutter type model for submarine avalanches and generated tsunami. J. Comput. Phys., 227(16), 77207754

[10] Fischer J-T, Kowalski J and Pudasaini SP (2012) Topographic curvature effects in applied avalanche modeling. Cold Regions Science and Technology, 74-75: 21-30 (doi:10.1016/j.coldregions.2012.01.005)

[11] Gray JMNT, Wieland M and Hutter K (1999) Gravity-driven free surface flow of granular avalanches over complex basal topography. Proc. R. Soc. A, 455, 1841-1874

[12] Hutter K, Svendsen B and Rickenmann D (1996) Debris flow modelling review. Continuum Mech. Thermo. Dyn., 8, 1-35

[13] Iverson RM (1997) The physics of debris flows. Rev. Geo-phys., 35(3), 245-296

[14] Iverson RM and Denlinger RP (2001) Flow of variably fluidized granular masses across threedimensional terrain: 1. Coulomb mixture theory. J. Geophys. Res., 106(B1), 537-552

[15] Jiang GS and Tadmor E (1998) Non-oscillatory central schemes for multi-dimensional hyperbolic conservation laws. SIAM, J. Sci. Comput., 19, 1892-1917

[16] Kafle J, Kattel P, Pokhrel PR, Khattri KB, Gurung DB and Pudasaini SP (2013) Dynamic interaction between a two-phase submarine landslide and a fluid reservoir. Int. J. Lsld. Env., 1(1), 35-36 (2013)

[17] Kafle J (2014) Dynamic interaction between a two-phase submarine landslide and a fluid reservoir. M. Phil. Dissertation, Kathmandu University, School of Science, Dhulikhel, Kavre, Nepal 
Journal of Nepal Mathematical Society (JNMS), Vol. 2, Issue 2 (2019); J. Kafle, P. Kattel

[18] Kafle J, Pokhrel PR, Khattri KB, Kattel P, Tuladhar BM and Pudasaini SP (2016) Landslide-generated tsunami and particle transport in mountain lakes and reservoirs. Annals of Glaciology, 57(71), 232-244 (doi: 10.3189/2016AoG71A034)

[19] Kafle J and Tuladhar BM (2018) Landslide-water interaction for partially submerged landslide. Journal of Nepal Mathematical Society, 1(1), 22-29

[20] Kafle J, Kattel P, Mergili M, Fischer J-T and Pudasaini SP (2019) Dynamic response of submarine obstacles to two-phase landslide and tsunami impact on reservoirs. Acta Mechanica, 230, 3143-3169 (doi : 10.1007/s00707-019-02457-0)

[21] Kattel P (2014) Dynamics of quasi-three-dimensional and two-phase mass flows. M. Phil. Dissertation, Kathmandu University, School of Science, Dhulikhel, Kavre, Nepal

[22] Kattel P, Khattri KB, Pokhrel PR, Kafle J, Tuladhar BM, and Pudasaini SP (2016) Simulating glacial lake outburst floods with a two-phase mass flow model. Annals of Glaciology, 57(71), 349-358 (doi: 10.3189/2016AoG71A039)

[23] Kattel P and Tuladhar BM (2018) Interaction of two-phase debris flow with lateral converging shear walls. Journal of Nepal Mathematical Society, 1(2), 40-52

[24] Kattel P, Kafle J, Fischer J-T, Mergili M, Tuladhar BM and Pudasaini SP (2018) Interaction of twophase debris flow with obstacles. Engineering Geology, 217:242-197 (doi: 10.1016/j.enggeo.2018.05.023)

[25] Lie KA and Noelle S (2003) An improved quadrature rule for the flux-computation in staggered central difference schemes in multidimensions. J. Sci. Comput., 18, 69-81

[26] Mergili M, Fischer J-T, Krenn J and Pudasaini SP (2017) r.avaflow v1, an advanced open-source computational framework for the propagation and interaction of two-phase mass flows. Geosci. Model Dev., 10(2), 553-569 (doi:10.5194/gmd-10-553-2017)

[27] Mergili M, Emmer A, Juřicová A, Cochachin A, Fischer J-T, Huggel C and Pudasaini SP (2018a) How well can we simulate complex hydro-geomorphic process chains? The 2012 multi-lake outburst flood in the Santa Cruz Valley (Cordillera Blanca, Peru). Earth Surf. Process. Landf., 43, 1373-1378 (doi.org/10.1002/esp.4318)

[28] Mergili M, Frank B, Fischer J-T, Huggel C and Pudasaini SP (2018b) Computational experiments on the 1962 and 1970 mass flow events at Nevado Huascaran (Peru) with r.avaflow: Lessons learned for predictive mass flow simulations. Geomorphology, 322, 15-28.

[29] Nessyahu H and Tadmor E (1990) Non-oscillatory central differencing for hyperbolic conservation laws. J. Comput. Phys., 87(2), 408-463

[30] Pitman EB and Le L (2005) A two-fluid model for avalanche and debris flows. Phil. Trans. R. Soc. A, 363(3), 1573-1601

[31] Piton G and Recking A (2016) Design of sediment traps with open check dams. I: Hydraulic and deposition Processes. Journal of Hydraulic Engineering (doi: 10.1061/(ASCE)HY.1943-7900.0001048)

[32] Pudasaini SP and Hutter K (2007) Avalanche Dynamics: Dynamics of Rapid Flows of Dense Granular Avalanches. Springer, Berlin, New York

[33] Pudasaini SP (2012) A general two-phase debris flow model. J. Geophysics. Res., 117, F03010 (doi:10.1029/2011JF002186)

[34] Pudasaini SP (2014) Dynamics of submarine debris flow and tsunami. Acta Mechanica, 225, 2423-2434 (doi: 10.1007/s00707-014-1126-0)

[35] Qiao C, Ou G and Pan H (2018) Numerical modelling of the long runout character of 2015 Shenzhen landslide with a general two-phase mass flow model. Bulletin of Engineering Geology and the Environment (doi:org/10.1007/s10064-018-1329-z) 
[36] Savage SB and Hutter K (1989) The motion of a finite mass of granular material down a rough incline. J. Fluid Mech., 199, 177-215

[37] Takahashi T (2007) Debris Flow: Mechanics, Prediction and Countermeasures. Taylor and Francis, New York

[38] Tai Y-C, Gray JMNT, Hutter K and Noelle S (2001) Flow of dense avalanches past obstructions. Annals of Glaciology, 32, 281-284

[39] Tai Y-C, Noella S, Gray JMNT and Hutter K (2002) Shock-capturing and front tracking methods for granular avalanches. J. Comput. Phys., 175(1), 269-301

[40] Viroulet S, Baker JL, Edwards AN, Johnson CG, Gjaltema C, Clavel P and Gray JMNT (2017) Multiple solutions for granular flow over a smooth two-dimensional bump. J. Fluid Mech., 815, 77-116 (doi.org/10.1017/jfm.2017.41)

[41] von Boetticher A, Turowski JM, McArdell BW, Rickenmann D and Kirchner JW (2017) DebrisInterMixing-2.3: a finite volume solver for three-dimensional debris-flow simulations with two calibration parameters-Part 2: Model validation. Geosci. Model Dev., 10, 3963-3978 (http: 10.5194/gmd$10-2017-25)$

[42] Wang X, Morgenstern NR and Chan DH (2010) A model for geotechnical analysis of flow slides and debris flows. Can. Geotech. J., 47, 1401-1414 (doi:10.1139/T10-039)

[43] Wawra M (2010) Numerical modelling of snow avalanches: Interaction between granular flow and obstruction, PhD Thesis, University of Soil Culture, Vienna

[44] Xie T, Yang H, Wei F, Gardner JS, Dai Z and Xie X (2014) A new water-sediment separation structure for debris flow defense and its model test. Bull. Eng. Geol. Environ., 73, 947-958 (doi: 10.1007/s10064014-0585-9)

[45] Xiao L, Ward S and Wang J (2015) Tsunami Squares Approach to Landslide-Generated Waves: Application to Gongjiafang Landslide, Three Gorges Reservoir, China. Pure Appl. Geophys. (doi: $10.1007 / \mathrm{s} 00024-015-10)$ 